Microbiology and Immunology, University of Melbourne, at the Peter Doherty Institute for Infection and Immunity, Vic, Australia; ${ }^{4}$ Menzies School of Health Research, Darwin, NT, Australia; "these authors contributed equally

Contact Dr Sadid F. Khan.

E-mail: sadid.khan@gmail.com

1. Ku HY, Chuang YC, Yu WL Clinical spectrum and molecular characteristics of Klebsiella pneumoniae causing community-acquired extrahepatic abscess. J Microbiol Immunol Infect 2008; 41: 311-7.

2. Liu KH, Lee HC, Chuang YC, et al. Prostatic abscess in southern Taiwan: another invasive infection caused predominantly by Klebsiella pneumoniae. J Microbiol Immunol Infect 2003; 36: 31-6.

3. Wyres KL, Wick RR, Gorrie C, et al. Identification of Klebsiella capsule synthesis loci from whole genome data. Microb Genom 2016; 2: e000102.

4. Wick RR, Heinz E, Holt KE, Wyres KL. Kaptive Web: user-friendly capsule and lipopolysaccharide serotype prediction for Klebsiella genomes. J Clin Microbiol 2018; 56: e197. 18.

5. Lam M, Wick R, Wyres K, et al. Genetic diversity, mobilisation and spread of the yersiniabactin-encoding mobile element ICEKp in Klebsiella pneumoniae populations. Microb Genom 2018; 4: e000196.

6. Lam MMC, Wyres KL, Judd LM, et al. Tracking key virulence loci encoding aerobactin and salmochelin siderophore synthesis in Klebsiella pneumoniae. Genome Med 2018; 10: 77.

7. Diancourt L, Passet V, Verhoef J, Grimont PA, Brisse S. Multilocus sequence typing of Klebsiella pneumoniae nosocomial isolates. J Clin Microbiol 2005; 43: 4178-82.

8. Hooton TM, Stamm WE. Diagnosis and treatment of uncomplicated urinary tract infection. Infect Dis Clin N Am 1997; 11: 551-81.

9. Arcilla MS, van Hattem JM, Haverkate MR, et al. Import and spread of extended-spectrum B-lactamase-producing Enterobacteriaceae by international travellers (COMBAT study): a prospective, multicentre cohort study. Lancet Infect Dis 2017; 17: 78-85.

10. Gorrie CL, Mirceta M, Wick RR, et al. Gastrointestinal carriage is a major reservoir of Klebsiella pneumoniae infection in intensive care patients. Clin Infect Dis 2017; 65: 208-15.

11. Roberts MJ, Parambi A, Barrett L, et al. Multifocal abscesses due to multiresistant Escherichia coli after transrectal ultrasound-guided prostate biopsy. Med J Aust 2013; 198: 282-4.

12. Russo TA, Marr CM. Hypervirulent Klebsiella pneumoniae. Clin Microbiol Rev 2019; 32: e1-9.

DOI: https://doi.org/10.1016/j.pathol.2020.10.011

\section{SMOFlipid causing spuriously high serum total bilirubin in an extremely premature neonate}

Sir,

Neonatal jaundice caused by unconjugated bilirubin is a common problem in neonates. ${ }^{1}$ Bilirubin is a product of heme breakdown through a cascade of enzyme-catalysed reactions in the reticuloendothelial system. ${ }^{2}$ In utero the fetus maintains a high haemoglobin concentration to effectively extract oxygen from maternal blood which has relatively low oxygen tension. Once ex utero this requirement falls significantly with the availability of abundant oxygen through breathing ambient air reducing the need for elevated haemoglobin. This, coupled with the shorter fetal erythrocyte lifespan, produces an excess load of haemoglobin, which is metabolised into bilirubin in the immediate post-natal period.

However, the high bilirubin load can easily overwhelm the immature excretory system, predisposing neonates to developing jaundice in the first week of life. Unconjugated, free bilirubin can cross the immature neonatal blood brain barrier causing selective damage of the central nervous system.
With acute bilirubin toxicity in neonates, there is a progression of symptomatology beginning with lethargy and decreased feeding, and progressing to variable changes in tone, high-pitched cry, seizures, and even death.

Here, we describe a case of an extremely premature female baby with a progressive rise in serum bilirubin, which could not be accounted for by clinical progression and whereby the serum bilirubin values measured by two different laboratory methods were vastly divergent. This case highlights the limitations of standard laboratory methods in measuring bilirubin for extremely premature infants and the importance of clinical correlation and communication between disciplines in reaching a definite diagnosis to guide management options.

The patient was an extremely preterm female baby of about 24 weeks of gestation. Her birthweight was less than $500 \mathrm{~g}$. She was delivered via emergency caesarean section due to worsening maternal pre-eclampsia. After initial resuscitation efforts, the baby was admitted into the neonatal intensive care unit (NICU). In the NICU, she received total parenteral nutrition, which contained $1-2 \mathrm{~g} / \mathrm{kg} /$ day of lipids (SMOFlipid, an emulsion of fish oil, medium chain fatty acids, olive oil, soybean oil). SMOFlipid is given as a source of calories and essential fatty acids for parenteral nutrition to premature babies by day $1-2$ of life because they cannot meet their nutritional needs using the enteral route in early days of life.

The patient's daily serum total bilirubin concentrations were monitored with heel prick blood sampling and direct spectrophotometry bilirubinometer (Unistat Bilirubinometer; Reichert Technologies, USA). At 24 hours of life, her bilirubin was $86 \mu \mathrm{mol} / \mathrm{L}$ (Fig. 1); hence, phototherapy was started according to hour-specific phototherapy threshold (80 $\mu \mathrm{mol} / \mathrm{L}$ ) for extremely low birth weight babies. ${ }^{1}$ There were no other risk factors for jaundice as she was not glucose-6phosphate dehydrogenase deficient and there was no evidence of $\mathrm{ABO}$ blood group incompatibility.

Eight hours after starting phototherapy at 30 hours of life, her bilirubin concentration was $196 \mu \mathrm{mol} / \mathrm{L}$. Her phototherapy was intensified to 6 phototherapy units with exposure of her entire body surfaces. At 43 hours of life, despite being on intensive phototherapy, her serum bilirubin increased further to $220 \mu \mathrm{mol} / \mathrm{L}$, exceeding the recommended level of exchange transfusion $(170 \mu \mathrm{mol} / \mathrm{L}){ }^{1}$ To investigate the worsening hyperbilirubinaemia, a complete blood count and liver function test were performed. Simultaneously, another heel prick blood sample was collected for testing on the bilirubinometer.

Her bilirubin measured by bilirubinometer was elevated at $293 \mu \mathrm{mol} / \mathrm{L}$. However, the corresponding bilirubin from the liver panel was abnormally low at $15 \mu \mathrm{mol} / \mathrm{L}$ (term day 3 reference intervals $75-120 \mu \mathrm{mol} / \mathrm{L}$; measured using AU58000, Beckman Coulter, USA). Aspartate aminotransferase (182 U/L; reference 30-160 U/L) and lactate dehydrogenase (5915 U/L; reference: 590-2300 U/L) were elevated. Complete blood count showed normochromic, normocytic anaemia (haemoglobin $100 \mathrm{~g} / \mathrm{L}$; reference 120$180 \mathrm{~g} / \mathrm{L})$, and high reticulocyte count $\left(132 \times 10^{9} / \mathrm{L}\right)$. Presence of anaemia, high lactate dehydrogenase measurement, and high reticulocyte count indicated haemolysis. Her white cell count and C-reactive protein, total protein, albumin, alanine aminotransferase, and renal function were normal.

Treatment for hyperbilirubinaemia, either intensive phototherapy or exchange transfusion, should always be 


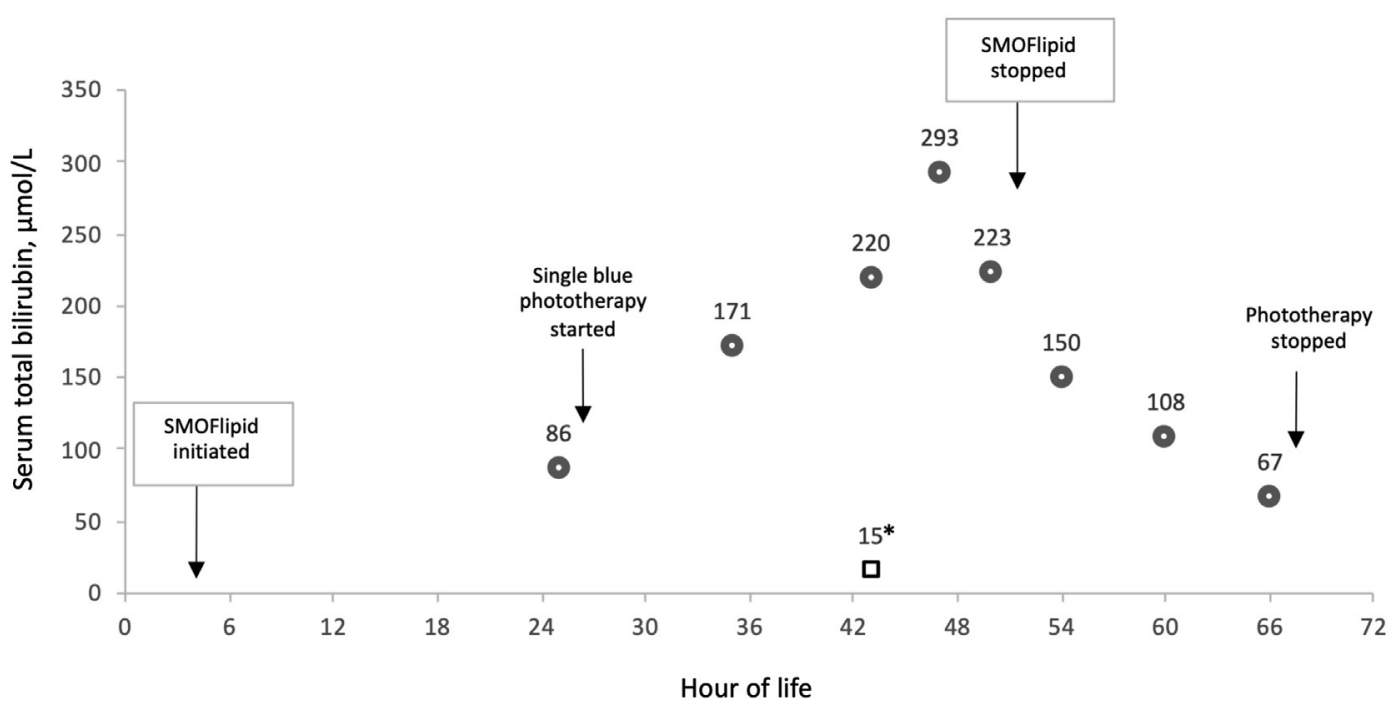

Fig. 1 Trend of bilirubin concentration over the first 70 hours of life. The bilirubin results represented by circles were measured by direct spectrophotometry bilirubinometer (Unistat Bilirubinometer; Reichert Technologies, USA), whereas the bilirubin result $(*)$ represented by an open square was measured by AU58000 analyser (Beckman Coulter, USA).

considered carefully as it can be potentially harmful in extremely premature babies. This is because intensive phototherapy can cause fluid loss and damage the fragile skin, while exchange transfusion is a high-risk procedure for an extremely premature baby that may result in significant haemodynamic instability leading to intraventricular haemorrhage in the brain or sepsis. Therefore, it needs to be performed after careful consideration of the clinical status and given bilirubin value. In this baby, there was no clinical sign of visible jaundice or kernicterus despite the progressive rise of serum bilirubin. Consequently, exchange transfusion was considered but not carried out as the progressive rise of bilirubin cannot be accounted for by the clinical progression.

Lipid infusion was stopped once the discrepancy in bilirubin values were made known to the neonatology team by the laboratory medicine team (Fig. 1). The baby remained well with no further rise in serum bilirubin after the lipid infusion and intensive phototherapy was stopped.

Bilirubin is most commonly measured by the diazo method, used in the AU5800 analyser, where it reacts with the diazonium salt in acidic conditions to form diazobilirubin (a red azo dye). Accelerators are added to complete the diazo reaction with the poorly soluble unconjugated bilirubin. The colour intensity of azo dye formed is monitored photometrically and is directly proportional to the concentration of total bilirubin in the sample. Haemoglobin interferes with diazo method through oxidative destruction of bilirubin or spectral interference from oxyhaemoglobin. ${ }^{2}$ The interference can be positive or negative and is highly sensitive to variation in reagents, dilutions and reaction timing. Generally, lipaemia does not interfere with the diazo method but it may occasionally be affected by paraproteins. ${ }^{6}$

Our in-house evaluation previously established strong negative interference on the AU5800 analyser in the presence of moderate haemolysis (data not shown), which explains the spuriously low bilirubin measurement in this baby. Ordinarily, the haemolysis would have been detected by the automated analyser as elevated haemolysis index and prevented the reporting of the spuriously low bilirubin. In this instance, as the baby was extremely small there was inadequate serum to determine the serum indices. Moreover, neonatal and infant samples are often haemolysed during the sample collection procedure. This can pose a problem for bilirubin measurement using diazo methods.

On the other hand, direct spectrophotometric measurement of bilirubin absorbance at $454 \mathrm{~nm}$, followed by subtraction of absorbance at $528 \mathrm{~nm}$ can eliminate the effect of haemolysis as haemoglobin absorbs equally at both $454 \mathrm{~nm}$ and $528 \mathrm{~nm}$. This method is particularly suited for neonatal testing as it requires a very small sample volume. However, other

Table 1 Interference of SMOFlipid emulsion on total bilirubin measurement by diazo method and direct spectrophotometry method

\begin{tabular}{lcccc}
\hline $\begin{array}{l}\text { Pooled neonatal } \\
\text { serum, } \mu \mathrm{L}\end{array}$ & SMOFlipid, $\mu \mathrm{L}$ & $\begin{array}{c}\text { Total bilirubin, } \\
\mu \mathrm{mol} / \mathrm{L} \text { (diazo method) }\end{array}$ & $\begin{array}{c}\text { Direct bilirubin, } \mu \text { mol/L } \\
\text { (diazo method) }\end{array}$ & $\begin{array}{c}\text { Total bilirubin, } \mu \mathrm{mol} / \mathrm{L} \\
(\text { direct spectrophotometry) }\end{array}$ \\
\hline 150 & 0 & 226 & 14 & 241 \\
150 & 5 & 228 & 15 & 408 \\
150 & 10 & 230 & 14 & 508 \\
150 & 20 & 225 & 15 & 759 \\
150 & 30 & 224 & 16 & $>11.4$ \\
150 & 40 & 232 & 15 & $>887$ \\
& & & $>11.4$ \\
\hline
\end{tabular}


pigments such as carotenoids can cause spectral interference at $454 \mathrm{~nm}$, limiting this method to neonates who have reached 2-3 weeks post-menstrual age.

Another routine method for bilirubin measurement is the use of vanadate oxidase whereby bilirubin (yellow) is oxidised to biliverdin (green) by vanadate in acidic conditions. ${ }^{6}$ The decrease in yellow absorbance is monitored and proportional to concentration of bilirubin. This method is usually adopted as an open channel reagent and is not sensitive to haemoglobin or lipaemic interference. ${ }^{6}$ However, this reference method is not available for the routine clinical laboratory as it requires the use of reverse phase high performance liquid chromatography.

In this baby, who was given SMOFlipid, the serum was grossly lipaemic when assessed visually (corresponding with triglycerides measurement of $>11.4 \mathrm{mmol} / \mathrm{L}$ ). The impact of lipaemic interference has been demonstrated from another form of intravenous emulsion (Lipofundin, a fat emulsion on the basis of medium and long chain triglycerides) ${ }^{8}$ but not SMOFlipid. As lipaemic interference is dependent on the size and composition of the lipid particles, ${ }^{9}$ increasing amounts of SMOFlipid were spiked into pooled neonatal serum and total bilirubin was measured using diazo and direct spectrophotometric methods in the laboratory for comparison (Table 1).

While the direct spectrophotometric method showed a dose-dependent positive interference, which explained the spurious gross hyperbilirubinaemia in this baby (Table 1), the diazo method was unaffected by SMOFlipid but affected by haemolysis resulting in low bilirubin concentration as measured by AU5800 analyser. Typically, the spuriously high bilirubin concentration is expected to decrease rapidly once the lipid infusion is stopped (Fig. 1). However, it did not happen in this case, as extremely premature babies have slower clearance of lipids as compared to adults who typically clear the infused lipids after 5-6 hours. ${ }^{10}$

In conclusion, lipid interference can cause high bilirubin value as measured by bilirubinometer, and presence of haemolysis can cause low bilirubin value as measured by diazo method. The susceptibility to interference should be considered when selecting and interpreting bilirubin results based on clinical situation. Laboratories serving the neonatal population should verify the haemoglobin and lipaemic interferences on their bilirubin measurement method and communicate the limitations with neonatologists. Prompt communication between neonatal and laboratory medicine teams is critical in deciding optimum management in this therapeutic dilemma and to avoid potentially harmful highrisk procedures.

Acknowledgement: We thank the staff of Department of Laboratory Medicine, National University Hospital, Singapore, for performing the tests.

Conflicts of interest and sources of funding: The authors state that there are no conflicts of interest to disclose.

\section{Jia Ming Low ${ }^{1}$, Tze Ping Loh $^{2}$, Zubair Amin ${ }^{1}$}

${ }^{1}$ Department of Neonatology, Khoo Teck Puat-National University Children Medical Institute, Singapore; ${ }^{2}$ Department of Laboratory Medicine, National University Hospital, Singapore

Contact Dr Tze Ping Loh.

E-mail: tploh@hotmail.com

1. National Collaborating Centre for Women's and Children's Health Neonatal jaundice. Clinical guideline May 2010. Cited 20 Apr 2020 https://www.nice.org.uk/guidance/cg98/evidence/full-guideline-245411 821

2. Kirk JM. Neonatal jaundice: a critical review of the role and practice of bilirubin analysis. Ann Clin Biochem 2008; 45: 452-62.

3. Shapiro MS. Definition of the clinical spectrum of kernicterus and bilirubin-induced neurologic dysfunction (BIND). J Perinatol 2005; 25: 54-9.

4. Shapiro SM. Chronic bilirubin encephalopathy: diagnosis and outcome. Semin Fetal Neonatal Med 2010; 15: 157-63.

5. Bhutani VK, Wong RJ. Bilirubin-induced neurologic dysfunction. Semin Fetal Neonatal Med 2015; 20: 1-64.

6. Dhungana N, Morris C, Krasowski MD. Operational impact of using a vanadate oxidase method for direct bilirubin measurements at an academic medical center clinical laboratory. Pract Lab Med 2017; 8: 7785 .

7. Kazmierczak SC, Robertson AF, Catrou PG, et al. Direct spectrophotometric method for measurement of bilirubin in newborns: comparison with HPLC and an automated diazo method. Clin Chem 2002; 48 1096-7.

8. Hsueh JT, Hwang KS, Pai L, et al. Effects of Lipofundin ${ }^{\circledR}$ on the measurement of total bilirubin by spectrophotometry. J Steroids Horm Sci 2011; 4: 2 .

9. Nikolac N. Lipemia: causes, interference mechanisms, detection and management. Biochem Med (Zagreb) 2014; 24: 57-67.

10. Food and Drug Administration (FDA). Highlights of prescribing information SMOFlipid. Cited 20 Apr 2020. https://www.accessdata.fda.gov/ drugsatfda_docs/label/2016/207648lbl.pdf

DOI: https://doi.org/10.1016/j.pathol.2020.09.023 